\title{
Artificial Neural Network Based Automatic Face Model Generation System from Only One Fingerprint
}

\author{
Seref Sagiroglu ${ }^{1}$ and Necla Ozkaya ${ }^{2}$ \\ ${ }^{1}$ Gazi University, Engineering and Architecture Faculty, Computer Engineering Department, \\ 06570 Ankara, Turkey \\ ${ }^{2}$ Erciyes University, Engineering Faculty, Computer Engineering Department, \\ 38039, Kayseri, Turkey \\ ss@gazi.edu.tr, neclaozkaya@erciyes.edu.tr
}

\begin{abstract}
Biometrics technology has received increasingly more attention during the last three decades. Since the performance of biometric systems has reached a satisfactory level for applications, a number of biometric features have been deeply studied, tested and successfully deployed in applications. Relationships among biometric features have not been studied so far. This study focuses on analysing the existence of any relationships among fingerprints and faces. For doing that an intelligent system based on artificial neural networks for generating face models including eyes, nose, mouth, ears and face border from only one fingerprint with the errors among 2.0-12.9 \% was developed. Experimental results have shown that there are close realitionships among fingerprints and faces and it is possible to generate faces from only one fingerprint image without knowing any information about faces. Although the proposed system is an initial study and it is still under development, the results are very encouraging and promising for the future developments and applications.
\end{abstract}

Keywords: Biometrics, artificial neural network, intelligent systems, fingerprint identification, face recognition.

\section{Introduction}

Biometrics is a science that extracts the physical or behavioral parameters of individuals with the aim of identification. Several personal biological characteristics such as fingerprint, face, iris, voice, or hand geometry are now used in biometric systems that are more reliable to identify people than traditional methods based on features that we have (key, card) or we know (password). Because a biometric system relies on specific characteristics of a particular person to register an identity. Therefore, it can differentiate between an authorized person and fraudulent impostor. So, any system assuring reliable person identification must necessarily involve a biometric component. Recently a number of biometric features have been successfully applied to the applications including information security, law enforcement, surveillance, forensics, smart cards, access control, time/place control points and computer networks etc. Furthermore various biometric devices and complete systems 
that provide business and benefit management solutions that using biometric based person identification systems have been produced and they are commercially available. In spite of all these developments in biometrics, there is no study on investigating relationships among the biometric features or obtaining one feature from another. It should be emphasized that most of the works in biometrics have been focused on how to improve the accuracy and processing time of the biometric systems, to design the more intelligent systems, and to develop more effective and robust techniques and algorithms [1], [2].

The aim of our study is intended to develop an automatic and intelligent system capable of generating the face of a person from only one fingerprint of the same person without having any priori knowledge about his or her face. In order to achieve that, an artificial neural network based intelligent face model generation system from only one fingerprint (fingerprint to face system: F2FS) has been developed and introduced in this study.

This paper is organized as follows. Section 2 briefly describes background information on biometrics, automatic fingerprint authentication systems (AFASs) and face recognition systems (FRSs). Section 3 basically introduces artificial neural networks (ANNs). Section 4 highlights the novelty of the proposed technique, introduces basic information, notation and performance metrics related to the F2FS and explains the various steps of the new approach. Section 5 demonstrates our experimental results. Finally, the proposed work is concluded and discussed in Section 6.

\section{Background}

In general, a biometric system gets a biometric data from a person, extracts a feature set from the acquired data, and compares this feature set against the template feature set in the database [3]. A biometric system works in four modes depending on the application status [4]: the enrolment, the verification, the identification and the screening. The enrolment is responsible for scanning, categorization and registration of the biometric characteristics. All other modes use the biometric data that was acquired to the system in the enrolment mode. In the verification, a person desired to be identified by submitting to the system a claim to an identity, usually via a magnetic card, login name, smart card etc., and the system either rejects or accepts the submitted claim of the identity at the end [5]. Commercial applications, such as physical access control, computer network logon, electronic data security, ATMs, credit-card purchases, cellular phones, personal digital assistants, medical records management and distance learning are samples of the verification applications [2], [4]. In the identification, the system identifies a person's identity without the person having to claim an identity or it fails if the person is not enrolled in the system database. The input and the output of the system are just a biometric feature and a combination of a list of identities and the scores indicating the similarity among two biometric features, respectively [5]. Welfare-disbursement, national ID cards, border control, voter ID cards, driver's license, criminal investigation, corpse identification, parenthood determination, missing children identification are from typical identification applications [2], [4]. In the screening, the results of determination 


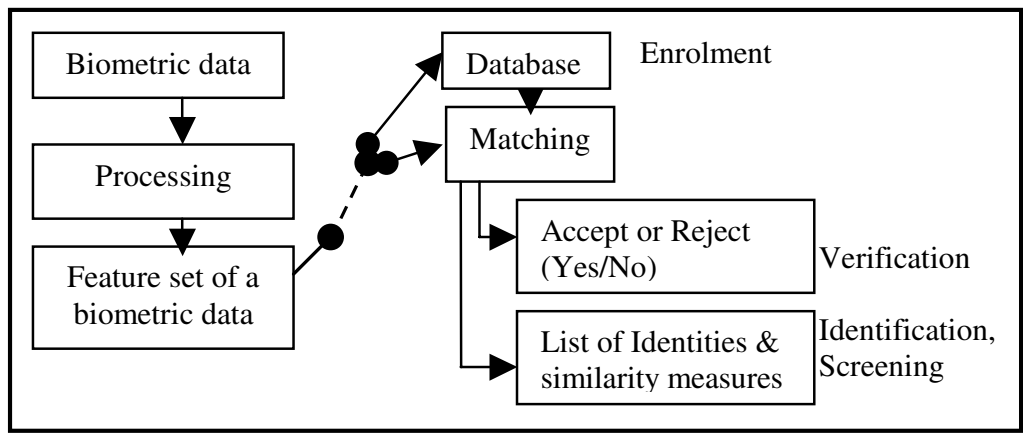

Fig. 1. A generic biometric system

whether a person belongs to a watch list of identities or not is displayed. Security at airports, public events and other surveillance applications are some of the screening examples [4], [6]. A generic biometric system is given in Fig. 1.

It is expected that a biometric system always takes the correct decision when a biometric feature is presented to the system. However, in practice a biometric system can make two basic types of errors: false match rate (FMR) and false non-match rate (FNMR) [1]. These errors generally used to show the accuracy and performance of the system in the literature. Nevertheless it is more informative to report the system accuracy in terms of a Receiver Operating Characteristic (ROC) curve that shows the system performance at all operating points [6].

\subsection{Automatic Fingerprint Authentication Systems}

Fingerprint is the most widely used biometric feature due to its uniqueness, immutability, reliability, permanence and universality [7]. It has a ridge-valley structure, core and delta points called singular points, end points and bifurcations called minutiaes. These structures are given in Fig. 2. Many approaches to AFASs have been presented in the literature [1], [2], [5], [7]-[19]. Yet, it is still an actively researched area.

The AFASs might be broadly classified as being minutiae-based, correlationbased and image-based systems [8]. A good survey about these techniques was given in [1]. The minutiae-based approaches rely on the comparisons for similarities and differences of the local ridge attributes and their relationships to make a personal

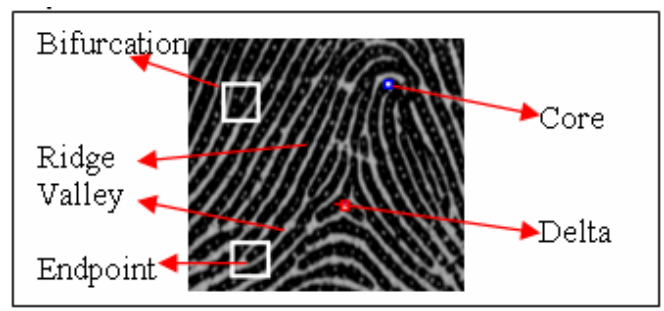

Fig. 2. Ridge-valley structure and features of a fingerprint 
identification [9]-[11]. They attempt to align two sets of minutiaes from two fingerprints and count the total number of matched minutiaes [4]. If minutaes and their parameters are computed relative to the singular points which are highly stable, rotation, translation and scale invariant, then these minutiaes will also become rotation, translation and scale invariant [5], [12]-[14]. Core points are the points where the innermost ridge loops are at their steepest. Delta points are the points from which three patterns deviate [13], [15], [16]. The general methods to detect the singular points are poincare-based methods [17], intersection-based methods [13] or filter-based methods [18]. Main steps of the operations in the minutiae-based AFASs are summarized as follows: selecting the image area, detecting the singular points, enhancing, improving and thinning the fingerprint image, extracting the minutiae points and calculating their parameters, eliminating the false minutiaes, properly representing the fingerprint images with their feature sets, recording the feature sets into a database, matching the feature sets, test and evaluating the system [19]. The results of these processes are given in Fig. 3, respectively. The performance of the minutiae-based techniques relies on the accuracy of all these processes. Especially the feature extraction and the use of sophisticated matching techniques to compare two minutiae sets often more affect the performance.

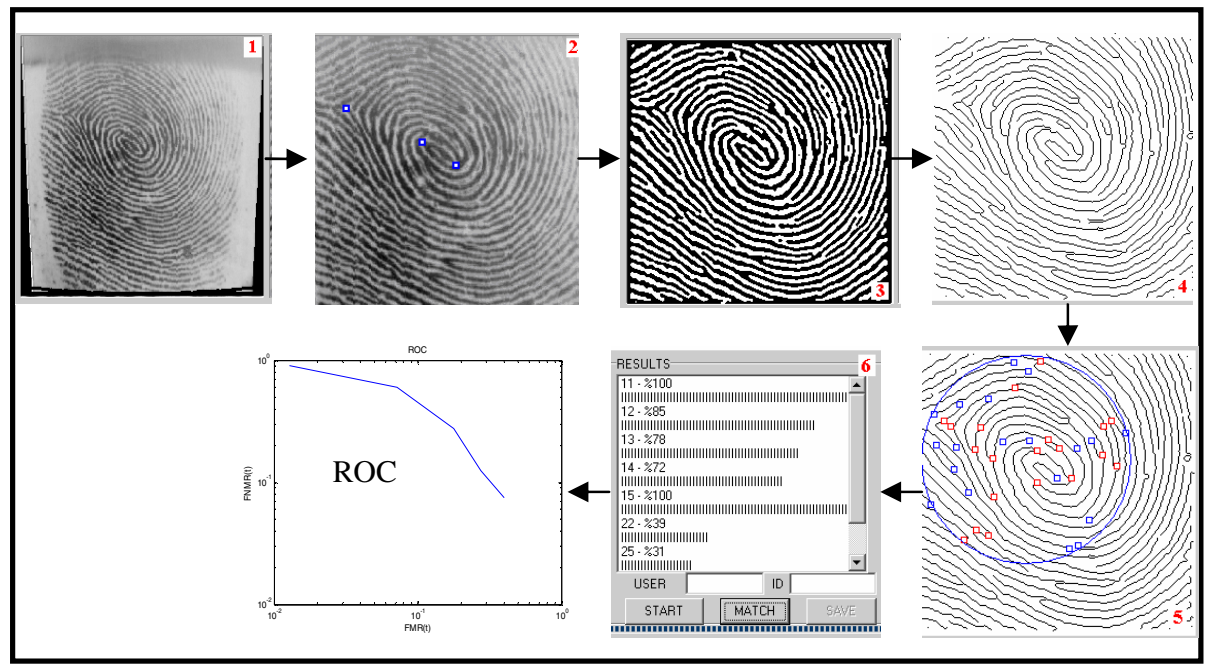

Fig. 3. Main operation steps of a minutiae-based AFAS [19]

In the correlation-based AFASs, global patterns of the ridges and valleys are compared to determine if the two fingerprints align. The template and query fingerprint images are spatially correlated to estimate the degree of similarity between them. The performance of the correlation-based techniques is affected by non-linear distortions and noise present in the image. In general, it has been observed that minutiae-based techniques perform better than correlation-based ones [20]. In the image-based approaches, the decision is made using the features that are extracted 
directly from the raw image that might be the only viable choice when image quality is too low to allow reliable minutiae extraction [8].

\subsection{Face Recognition Systems}

Face is probably the most common biometric characteristic used by humans to make personal recognition. Face recognition is an active area of research with several applications ranging from static to dynamic [9]. So, face recognition technology is well advanced. In general, a FRS consists of three main steps. These steps cover detection of the faces in a complicated background, localization of the faces followed by extraction of the features from the face regions and finally identification or verification tasks [21]. Face detection and recognition process is really complex and difficult due to numerous factors affecting the appearance of an individual's facial features such as 3D pose, facial expression, hair style, make-up, etc. [22]. In addition to these varying factors, lighting, background, scale, noise and face occlusion and many other possible factors make these tasks even more challenging [21]. The most popular approaches to face detection and recognition are based on either the location and shape of the facial attributes, such as the faces, eyebrows, nose, lips and chin and their spatial relationships or the overall analysis of the face images that represents a face as a weighted combination of a number of canonical faces [4], [21], [23]. Also many effective and robust methods for the face recognition have been proposed [2], [9], [21]-[25]. They are categorized as follows: Knowledge-based methods encode human knowledge of what constitutes a typical face. Feature invariant methods aim to find structural features that exist even when the pose, viewpoint or lighting conditions vary to locate faces. In template matching based methods several standard patterns of a face are used to describe the face as a whole or the facial features separately. Appearance-based methods operate directly on images or appearances of the face objects and process the images as two-dimensional holistic patterns [23].

\section{Artificial Neural Networks}

ANNs have been applied to solve many problems [26], [30]. Learning, generalization, less data requirement, fast computation, ease of implementation and software and hardware availability features have made the ANNs very attractive for many applications [27], [28]. These fascinating features have also made them popular in biometrics as well [23], [24], [28]-[30]. Multilayered perceptron (MLP) is one of the most used ANN architectures. Because of the MLP structure can be trained by many learning algorithms, it has been applied to a variety of problems successfully in the literature. The MLP structure consists of three layers: input, output and hidden layers. One or more hidden layers might be used. The neurons in the input layer can be treated as buffers and distribute $x_{i}$ input signals to the neurons in the hidden layer. The output of the each neuron $y_{j}$ in the hidden layer is obtained from sum of the multiplication of all input signals $x_{i}$ and weights $w_{j i}$ that follow all these input signals. The sum can be calculated as a function of $y_{j}$ and can be expressed as:

$$
y_{j}=f\left(\sum w_{j i} x_{i}\right)
$$


where $f$ can be a simple threshold function, a hyperbolic tangent or a sigmoid function. The outputs of the neurons in other layers are calculated in the same way. The weights are adapted with the help of a learning algorithm according to the error that can be calculated by subtracting the ANN output from the desired output. The ANNs might be trained with many different learning algorithms [28].

\section{Proposed ANN Based Intelligent Face Generation System}

Fingerprint and face recognition topics have received significantly increased attention due to possessing the merits of their reliability, performance and high accuracy. The proposed F2FS generates the face of a person from only one fingerprint of the same person without having any information about his or her face. It is thought that it will be a very interesting innovation to biometrics.

Implementation steps of the F2FS to establish a relationship among fingerprints and faces (Fs\&Fs) can be mentioned as follows:

1. A database consisted of Fs\&Fs was established.

2. Feature sets of Fs\&Fs were obtained.

3. Training and test data sets were established for ANN application.

4. Suitable ANN parameters were selected.

5. Randomly selected 80 of 120 data sets covering pairs of Fs\&Fs were used to train the ANN based F2FS.

6. Feature sets of test sets covering only fingerprints of remaining 40 people from the database were used to test the system.

7. In order to evaluate the accuracy of the F2FS, the test results were compared with their desired values against to a variety of state-of-the-art methods [1].

The proposed F2FS has constructed by appropriately combining a data enrolment module, a feature extraction module, an ANN module, an evaluation module and a face reconstruction module. The first module of the system which is called the data enrolment module helps store biometric data of individuals into the biometric system database. During this process, Fs\&Fs of an individual have been captured to produce a digital representation of the characteristics. Two types of biometric data are used in this study: Fs\&Fs. The second module of the system extracts discriminative feature sets from the acquired data. Extracting local and global feature sets of the fingerprints, which include fingerprint singularities, minutiae points and their parameters, is achieved. Similarly feature sets of the faces were obtained. The ANN module is used to analyze the existence of any relationship among Fs\&Fs. This part of the system was implemented with the help of 3-layered ANN structure that is trained with the scaled conjugate gradient (SCG) algorithm. The SCG algorithm is an ANN training algorithm that adjusts the weights and biases of an ANN structure according to its learning strategy. The SCG algorithm is based on conjugate directions. The details of SCG algorithm can be found in the references [31] and [32]. Sigmoid transfer function was used in the proposed study for generating the output of each neuron used in the structure. The ANN module has only a hidden layer with 200 neurons. The block diagram of the ANN Module is given in Fig. 4. 


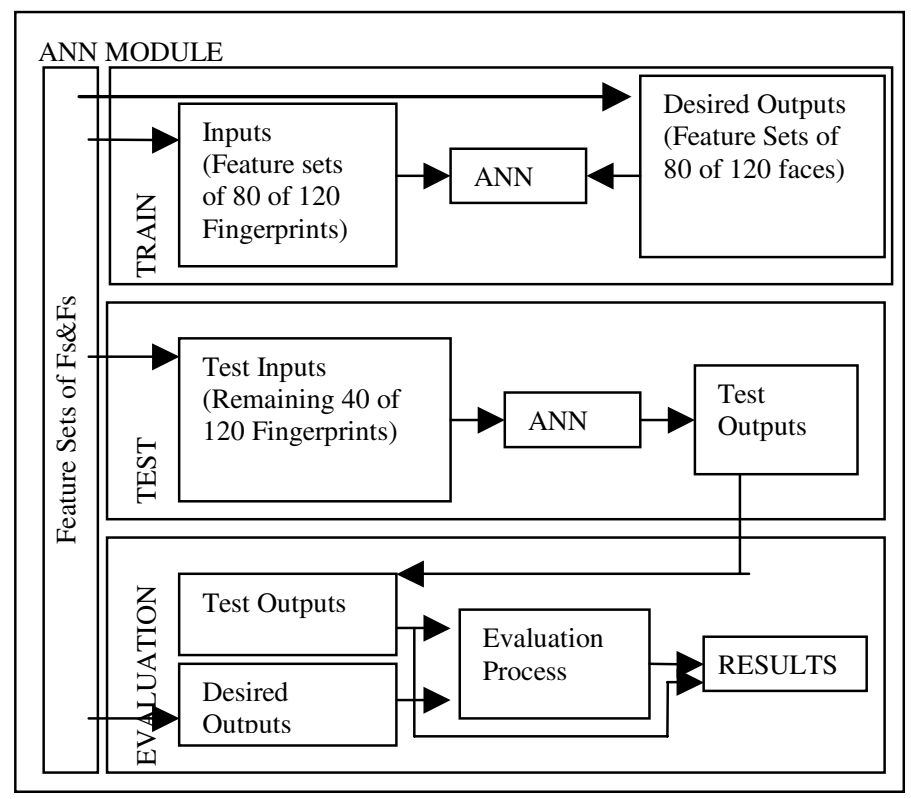

Fig. 4. The block diagram of the ANN Module

The ANN module is the most critical and important module of the system. Because, all modules of the system except the ANN module are on duty, either in preprocessing or post-processing of the main process that is done by the ANN module. So, if we investigate this module deeply, we can explain the working principles of the F2FS properly.

The ANN module operates its task in two stages: the training and the testing. In the training process, randomly selected input-output image sets covering Fs\&Fs belonging to the same people were used. The ANN structure and the training parameters were determined for achieving the training stage accurately. The training process is started with applying a person's fingerprint feature set to the system as an input and same person's face feature set as the desired output. The system achieves the training process with these feature sets according to the learning algorithm and the ANN parameters. Both fingerprint and face feature sets are required in the training. Only fingerprint feature sets are used in test. These fingerprints are unknown biometric data to the F2FS.

Producing the faces as close to the real one as possible is critical for this study. The traditional metrics of an ordinary biometric system are no longer appropriate to characterize the performance of the F2FS. In addition to the FMR-FNMR and the ROC curve representations, the results of the system were evaluated to give more prices perceptions to the researchers by considering the following metrics: mean squared error (MSE), sum squared error (SSE), average correlation, absolute percent error (APE) and mean APE [33]. In addition to these numerical evaluations, a visual evaluation platform was also created from face features by drawing the results of desired and actual outputs together. Moreover, another visual evaluation platform was 
also established by drawing the results of actual and desired outputs on the involved real face images of test people.

Consequently, for a more objective comparison, the performance and accuracy of the system have been evaluated and presented on the basis of the combination of these metrics for illustrating the qualitative properties of the proposed methods as well as a quantitative evaluation of their performance. The face reconstruction module facilitates the evaluation process, simplifies to understand the results and presents to the users to evaluate the results perceptionally. To achieve all these processes easily and efficiently, an automatic system has been proposed and a graphical interface was designed to achieve the results and the metrics in the expected form.

\section{Experimental Results}

The concept of generating faces from only one fingerprint is a novel and challenging idea to biometrics technology. The proposed ANN based face generation system from only one fingerprint that was discussed in previous section is implemented. A dedicated software has been developed to conduct the experiments easily and efficiently. In the experiments, a multimodal database having Fs\&Fs belonging to 120 people was established. The index finger of the right hand was used because of being the most used finger in AFASs.

In the training processes, 80 of 120 image sets were randomly selected from the database. The remaining 40 of 120 fingerprint images were used in the test. The experimental image sets used in test processes contain only fingerprint images of the test people and these data sets are unknown data for the system. The face images of the test people were also used for evaluation of the system's performance. The inputs and the outputs of the system were vectors sized 298 and 148, respectively. These vectors were the feature sets of fingerprints and faces, respectively. The feature vectors of fingerprints were computed using a SDK developed by Neurotechnologija. The reason of this preference was to prove the system's success with a known method for the F2FS. This software is known as an effective, robust and reliable AFAS in the field of biometrics and it uses a minutiae-based algorithm. Detailed explanation of its algorithms, detailed information of fingerprint feature sets and its storage format are given in [34].

To get the feature sets of the faces a feature-based approach has been selected from the face recognition literature and used by modifying fundamentally [35]. It can be explained the reason of this preference is that it is used a minutiae-based approach to get the feature sets of the fingerprints. Actually minutiae-based approaches rely on the physical features of the fingerprints. Therefore it is reasonable that the feature sets of both Fs\&Fs should be obtained in the same way. So, a feature-based approach was used to get the feature sets of the faces. A template was used for faces at the beginning to provide appropriate features to the ANN model.

In order to evaluate the performance of the system effectively, we have benchmarked our system against to the extra metrics in addition to the traditional evaluation metrics of biometric systems that include FMR-FNMR representation and ROC curve of the test results. The metrics MSE and SSE were computed before rescaling, while the other metrics MSE, SSE, average APE and average correlation 


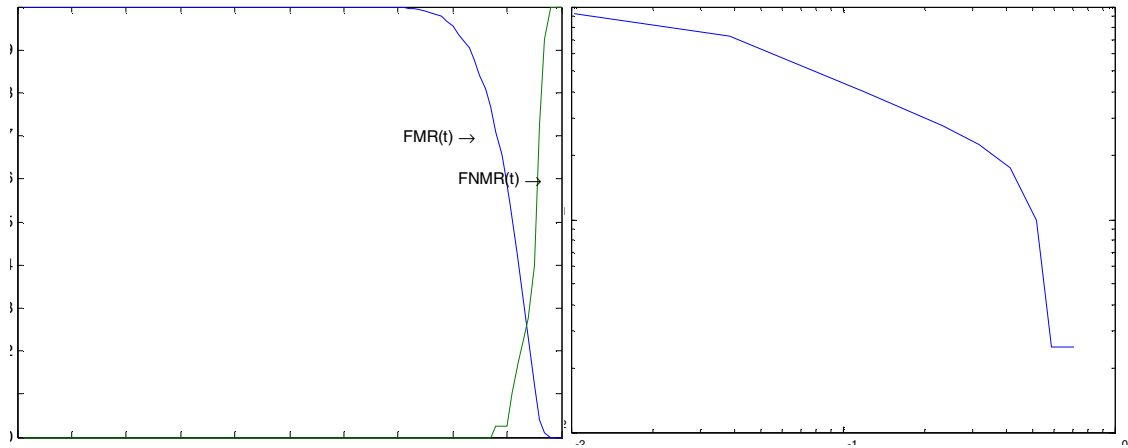

Fig. 5. FMR-FNMR representation and ROC curve of the test results

were calculated after rescaling as 8.8583e-004, 5.2441, 132.2764, 7.8308e+005, 5.43656 and 0.993698 , respectively. FMR-FNMR representation and ROC curve of the test results are given in Fig. 5.

These results indicated that the proposed system performed the tasks with high similarity measures to the desired values. For the purpose of more realistic and visual evaluation, 12 of 40 desired and achieved test results were drawn on the same platform as shown in Fig. 6. The same test results were shown on the real face images as given in Fig. 7. It needs to be emphasized that because of the page limitation, only 12 of 40 test results are given. However it is possible to show the overall system performance graphically for the all test results. The APE values belonging to all test results were demonsrated in Fig. 8.

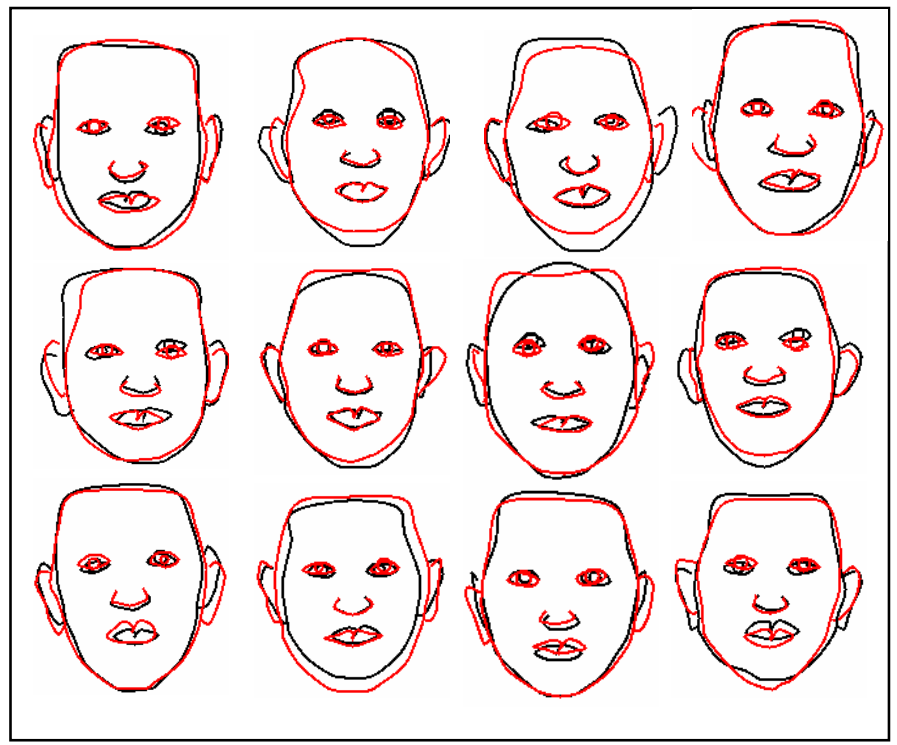

Fig. 6. Combined the test faces achieved from the F2FS and their desired values 


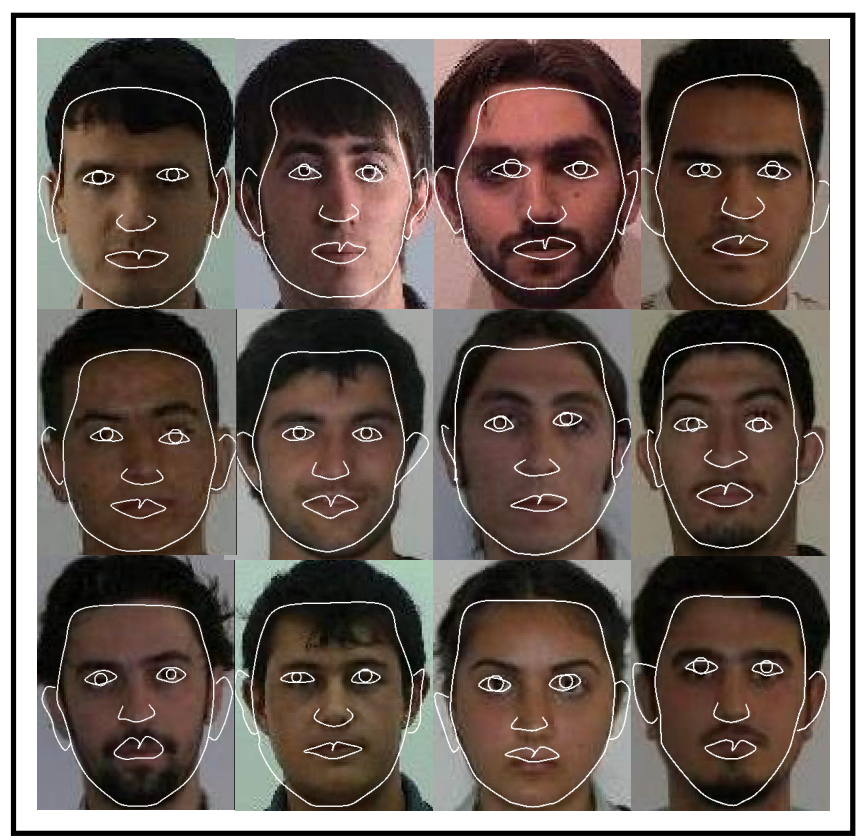

Fig. 7. Test faces obtained from the system are drawn on the real face images

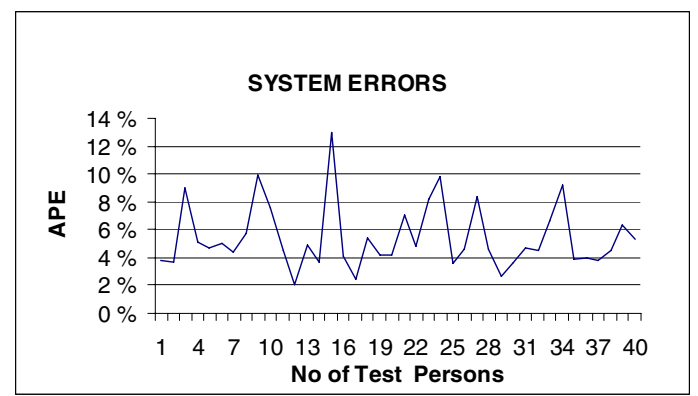

Fig. 8. APE values for all test results

Besides the numerical results indicated the system success clearly, graphical results also confirmed this success as well. As it can be seen from Figures 5-8, the system is very successful in achieving the faces according to each standard metric defined in the literature or extra metrics presented by us. Based on the observations, the fundamental novelty and diversity of the proposed approach over the most other studies in biometrics was to investigate the relationships between fingerprints and faces. In addition, the proposed system can be used for an intelligent and efficient translator that effectively converts a person's fingerprint to the face of the same person without any information about his or her face. 


\section{Conclusions and Future Work}

In this study, the existence of relationships among biometric features was experimentally illustrated. In addition, it is demonstrated that it is possible to achieve an unknown biometric feature from a known biometric feature, successfully.

It has a very simple structure. It is constructed by appropriately combining a data enrolment module, a feature extraction module, an ANN module, a test \& evaluation module and a face reconstruction module. In order to achieve the experiments easily and effectively, these modules were combined in the F2FS for generating the face models from fingerprints without any need of face information. Because of establishing the relationship among Fs\&Fs, the structure of the ANN module plays an important role in the system. The difficulty faced in the study was to explain the relationships among Fs\&Fs mathematically.

Finally it is hoped that this approach would lead to create new concepts, research areas, and especially new applications in the field of biometrics.

\section{References}

1. Maio, D., Maltoni, D., Jain, A.K., Prabhakar, S.: Handbook of fingerprint recognition. Springer, New York (2003)

2. Jain, L.C., Halici, U., Hayashi, I., Lee, S.B., Tsutsui, S.: Intelligent biometric techniques in fingerprint and face recognition. CRC press, New York (1999)

3. Jain, A.K., Ross, A., Prabhakar, S.: An introduction to biometric recognition. IEEE Trans. on Circuits and Systems for Video Technology 14(1), 4-19 (2004)

4. Jain, A.K., Ross, A., Pankanti, S.: Biometrics: a tool for information security. IEEE Trans. on Information Forensics and Security, 1(2), 125-143 (2006)

5. Jain, A.K., Hong, L., Pankanti, S., Bolle, R.: An identity authentication system using fingerprints. Proceedings of the IEEE 85(9), 1365-1388 (1997)

6. Jain, A.K., Pankanti, S., Prabhakar, S., Hong, L., Ross, A., Wayman, J.L.: Biometrics: A Grand Challenge. In: Proceedings of the Int. Conf. on Pattern Recognition, Cambridge, UK, August, vol. II, pp. 935-942 (2004)

7. Kovács-Vajna, Z.M.: A fingerprint verification system based on triangular matching and dynamic time warping. IEEE Trans. Pattern Anal. Mach. Intell. 22(11), 1266-1276 (2000)

8. Lumini, A., Nanni, L.: Two-class Fingerprint matcher. Pattern Recognition 39(4), 714-716 (2006)

9. Hong, L., Jain, A.: Integrating faces and fingerprints for personal identification. IEEE Trans. Pattern Analysis and Machine Intelligence 20(12), 1295-1307 (1998)

10. Jain, A.K., Hong, L., Bolle, R.: On-line fingerprint verification. IEEE Trans. on Pattern Analysis and Machine Intelligence 19(4), 302-314 (1997)

11. Zhou, J., Gu, J.: Modeling orientation fields of fingerprints with rational complex functions. Pattern Recognition 37(2), 389-391 (2004)

12. Hsieh, C.T., Lu, Z.Y., Li, T.C., Mei, K.C.: An Effective Method To Extract Fingerprint Singular Point. In: The Fourth Int. Conf./Exhibition on High Performance Computing in the Asia-Pacific Region, pp. 696-699 (2000)

13. Rämö, P., Tico, M., Onnia, V., Saarinen, J.: Optimized singular point detection algorithm for fingerprint images. In: Int. Conf. on Image Processing, pp. 242-245 (2001) 
14. Zhang, Q., Yan, H.: Fingerprint classification based on extraction and analysis of singularities and pseudo ridges. Pattern Recognition 11, 2233-2243 (2004)

15. Wang, X., Li, J., Niu, Y.: Definition and extraction of stable points from fingerprint images. Pattern Recognition 40(6), 1804-1815 (2007)

16. Li, J., Yau, W.Y., Wang, H.: Combining singular points and orientation image information for fingerprint classification. Pattern Recognition 41(1), 353-366 (2008)

17. Kawagoe, M., Tojo, A.: Fingerprint pattern classification. Pattern Recognition 17(3), 295303 (1984)

18. Nilsson, K., Bigun, J.: Localization of corresponding points in fingerprints by complex filtering. Pattern Recognition Lett. 24, 2135-2144 (2003)

19. Ozkaya, N., Sagiroglu, S., Wani, A.: An intelligent automatic fingerprint recognition system design. In: 5th Int. Conf. on Machine Learning and App., pp. 231-238 (2006)

20. Ross, A., Jain, A.K., Reisman, J.: A Hybrid Fingerprint Matcher. Pattern Recognition 36(7), 1661-1673 (2003)

21. Cevikalp, H., Neamtu, M., Wilkes, M., Barkana, A.: Discriminative common vectors for face recognition. IEEE Trans. on Pattern Analysis and Machine Intelligence 27(1), 4-13 (2005)

22. Bouchaffra, D., Amira, A.: Structural Hidden Markov Models for Biometrics: Fusion of Face and Fingerprint. Special Issue of Pattern Recognition Journal, Feature Extraction and Machine Learning for Robust Multimodal Biometrics (Article in press, 2007) (available online)

23. Li, S.Z., Jain, A.K.: Handbook of Face Recognition. Springer, NewYork (2004)

24. Yang, M.H., Kriegman, D.J., Ahuja, N.: Detecting faces in images: a survey. IEEE Trans. on Pattern Analysis and Machine Intelligence 1(24), 34-58 (2002)

25. Zhao, W., Chellappa, R., Phillips, P.J., Rosenfeld, A.: Face recognition: a literature survey. ACM Computing Surveys 35, 399-459 (2003)

26. Haykin, S.: Neural Networks: A Comprehensive Foundation. Macmillan College Publishing Company, New York (1994)

27. Sagiroglu, S., Beşdok, E., Erler, M.: Artificial intelligence applications in Engineering I: artificial neural networks. Ufuk publishing, Kayseri, Turkey (2003)

28. Sagar, V.K., Beng, K.J.A.: Hybrid Fuzzy Logic And Neural Network Model For Fingerprint Minutiae Extraction. In: Int. Conf. on Neural Netw., pp. 3255-3259 (1999)

29. Nagaty, K.A.: Fingerprints classification using artificial neural networks: a combined structural and statistical approach. Neural Networks 14, 1293-1305 (2001)

30. Maio, D., Maltoni, D.: Neural network based minutiae filtering in fingerprints. In: 14 th Int. Conf. on Pattern Recognition, pp. 1654-1658 (1998)

31. The Mathworks, Accelerating the Pace of Engineering and Science (2008), http:// www. mathworks.com/access/helpdesk/help/toolbox/nnet/nnet.html?/ access/helpdesk/help/toolbox

32. Moller, M.F.: A Scaled Conjugate Gradient Algorithm for Fast Supervised Learning. Neurall Networks 6, 525-533 (1993)

33. Novobilski, A., Kamangar, F.A.: Absolute percent error based fitness functions for evolving forecast models. In: FLAIRS Conf., pp. 591-595 (2001)

34. Biometrical and Artificial intelligence Technologies (2008), http: / /www.neurotechnologija.com/vf_sdk.html

35. Cox, I.J., Ghosn, J., Yianilos, P.N.: Feature-Based Face Recognition Using Mixture Distance. Computer Vision and Pattern Recognition, 209-216 (1996) 